\title{
Self- versus physician-collected samples for the follow-up of human papillomavirus-positive women in sub-Saharan Africa
}

This article was published in the following Dove Press journal: International Journal of Women's Health

\author{
Manuela Viviano ${ }^{1, *}$ \\ Phuong Lien Tran',* \\ Bruno Kenfack ${ }^{2}$ \\ Rosa Catarino' \\ Mohamed Akaaboune ${ }^{1, \dagger}$ \\ Liliane Temogne ${ }^{2}$ \\ Eveline Tincho Foguem ${ }^{2}$ \\ Pierre Vassilakos ${ }^{3}$ \\ Patrick Petignat ${ }^{1}$ \\ 'Gynecology Division, Geneva \\ University Hospitals, Geneva, \\ Switzerland; ${ }^{2}$ Department of \\ Biomedical Sciences, University \\ of Dschang, Dschang, Cameroon; \\ ${ }^{3}$ Geneva Foundation for Medical \\ Education and Research, Geneva, \\ Switzerland \\ *These authors contributed equally \\ to the work
}

†MA passed away on July 31,2017
Correspondence: Manuela Viviano Gynecology Division, Geneva University Hospitals, Boulevard de la Cluse 30, I 205 Geneva, Switzerland

Tel +4I 795532272

Email manuela.viviano@hcuge.ch
Introduction: Human papillomavirus (HPV) testing is a suitable tool for primary cervical cancer (CC) screening and follow-up in low-resource settings. Vaginal samples taken by women themselves (Self-HPV) are an interesting alternative to physician-performed sampling (Dr-HPV). Our aim was to assess the performance of Self-HPV and Dr-HPV at 6 and 12 months following a CC screening campaign.

Methods: This study was carried out at the Dschang District Hospital, Cameroon. Women aged 30-49 years were recruited in a CC screening campaign. HPV-positive women, of whom $2 / 3$ were treated with thermoablation because of abnormal results at baseline screening, were invited to participate in a follow-up study. Self- and Dr-HPV, as well as cytology, were performed at 6 and 12 months. HPV samples were analyzed using the Xpert HPV assay. Sensitivity and specificity for the detection of low-grade squamous intraepithelial lesion or worse and of high-grade squamous intraepithelial lesion or worse were calculated for Self-HPV and Dr-HPV, using cytology as the reference diagnosis.

Results: Overall, 188 HPV-positive women were invited to attend follow-up. The obtained follow-up visits' attendance was 154 (81.9\%) and 131 (69.7\%) at 6 and 12 months, respectively. While the overall performance of Dr-HPV at 6 months was slightly superior, Self-HPV showed an improved sensitivity for HSIL+ detection at 12 months when compared with Dr-HPV $(83.3 \%$ [95\% CI 41.8-98.9] versus 71.4\% [95\% CI 21.5-95.8], respectively). The overall HPV positivity agreement between Self- and Dr-HPV at 6 and 12 months corresponded to a $\kappa$ value of 0.62 and 0.52 , respectively. Among women treated with thermoablation $(\mathrm{n}=121)$ at baseline screening, Self-HPV was as sensitive as Dr-HPV, although less specific $(P=0.003)$.

Conclusion: Self-HPV is a valuable tool for the follow-up of HPV-positive women in lowresource settings. Larger, randomized trials are needed to confirm the validity of our findings. Keywords: human papillomavirus testing, self-sampling, cervical cancer, screening, follow-up

\section{Introduction}

Cervical cancer $(\mathrm{CC})$ is the fourth most common cancer in women worldwide, with $85 \%$ of cases occurring in developing countries. ${ }^{1}$ Persistent human papillomavirus (HPV) infection is the prerequisite for the development of cervical intraepithelial neoplasia (CIN) and CC. ${ }^{2}$ Following the trend of HPV infection over time and identifying persistent infections constitute one of the fundamental bases for CC prevention. ${ }^{3}$

In low-to-middle income countries (LMIC), visual inspection with acetic acid (VIA) and visual inspection with Lugol's iodine (VILI) are the recommended tools for primary $\mathrm{CC}$ screening. These methods require a pelvic examination and the presence of an experienced health-care professional. Follow-up for screen-positive women based 
on a pelvic examination is difficult to implement in LMIC, where the availability of resources is hard to ensure. ${ }^{4}$

It is paramount to develop a follow-up strategy that relies on economical and logistically simple procedures in low-income settings. The recent introduction of rapid polymerase chain reaction (PCR) tests makes it possible to detect viral infection within an hour of sample collection, thus enabling to further manage HPV-positive women on the same day. ${ }^{5}$ In addition, HPV testing can be performed on self-collected vaginal samples (Self-HPV) while guaranteeing the same diagnostic accuracy as physician-collected samples (Dr-HPV). ${ }^{6}$ By circumventing the need of a clinicbased pelvic examination, Self-HPV offers the opportunity to potentially break down some of the barriers to follow-up visits attendance. As the current evidence has been focusing primarily on the use of Self-HPV for primary CC screening, only a few data on the use of Self-HPV for the follow-up of HPV-positive women are available.

Considering the limited availability of resources and the high risk of loss to follow-up in LMIC, it is of utmost interest to develop an effective strategy for the detection of residual or recurrent disease while guaranteeing a high patient participation in care. Our aim was to compare the performance of Self-HPV and Dr-HPV for the follow-up of HPV-positive women at 6 and 12 months after baseline screening.

\section{Methods \\ Study setting}

This study took place at the Dschang District Hospital, which is located in Cameroon's Western region and counts $\sim 250,000$ inhabitants. In July 2015, all women aged 30-49 years were recruited in a CC screening campaign. Exclusion criteria were ongoing pregnancy and previous total hysterectomy. A total of 1,012 women were recruited in the study. The participants performed HPV self-sampling (SelfHPV) for primary screening. The samples were promptly analyzed using a point-of-care assay (GeneXpert ${ }^{\circledR} I V$; Cepheid, Sunnyvale, CA, USA). All women with a positive high-risk HPV test underwent a gynecological examination including VIA and VILI. When VIA revealed a pathological area, a biopsy of the suspected lesion was taken. If VIA revealed no pathological area, a 6 o'clock biopsy sample was taken. Endocervical brushing was performed on all HPV-positive women. Treatment, if needed, was performed using thermoablation. The HPV test, triage with VIA and VILI, and treatment were performed within the same day. Additionally, all study participants were asked to complete a questionnaire on sociodemographics as well as obstetric and gynecological histories.

\section{Study design}

Women positive for HPV at primary screening were asked to come for a follow-up visit at 6 and 12 months. The participants were called by the local medical personnel for their follow-up visits. Women were invited to perform Self-HPV using a dry swab, which was subsequently immersed in $5 \mathrm{~mL}$ of an $\mathrm{NaCl} 0.9 \%$ solution and vortexed for 10 seconds. One milliliter of this solution was then placed into a GeneXpert cartridge and run on the four-module GeneXpert machine. The physician also collected a sample for HPV testing and cytology. Cervical cells were collected using a Cervex-Brush Combi (Rovers, Oss, the Netherlands) as recommended by the European guidelines ${ }^{7}$ and, then, transferred into a BD SurePath ${ }^{\mathrm{TM}}$ collection vial containing a preservative fluid (BD, Franklin Lakes, NJ, USA).

The sample was vortexed for 10 seconds, and an aliquot of $1 \mathrm{~mL}$ was placed in the GeneXpert cartridge. Each sample was analyzed within 20 minutes from its collection. The rest of the sample was transported to Geneva, Switzerland, for cytological specimen analysis. All women, regardless of the HPV test result, underwent a pelvic examination with VIA and VILI. A biopsy and ECC sample were collected from women presenting with a pathological VIA, as well as from all the previously treated participants, to assess their disease status.

\section{HPV testing}

The GeneXpert HPV assay used for HPV testing consists of a real-time PCR that uses the detection of a human reference gene (hydroxymethylbilane synthase) and an internal probe check control for specimen adequacy. The probe check control was used to verify reagent rehydration, PCR tube filling in the cartridge, probe integrity, and dye stability. The Xpert test included reagents for the simultaneous detection of 14 high-risk HPV (hrHPV) genotypes (HPV-16, -18, -31, $-33,-35,-39,-45,-51,-52,-56,-58,-59,-66$, and -68$)$. The assay uses multiple fluorescent channels for the detection of individual HPV types, pooled HPV types, and the human reference gene. Each fluorescent channel has specific cutoff parameters for target detection and validity. If a sufficient amount of signal is detected for the human reference gene, the assay results are reported as positive. Additionally, HPV-16, pooled HPV-18/45, and pooled other hrHPV types detected by the assay are reported separately as either positive or negative. The test results are available within 50 minutes of the cartridge's introduction into the device.

\section{Cytology}

Thin-layer slides were prepared from the remaining in the preservative fluid sample using the BD Totalys ${ }^{\mathrm{TM}}$ SlidePrep 
processor (BD) located at Cytopath-Unilabs Laboratories, Geneva, Switzerland. All slides were read by a cytopathologist (PV) and were classified according to the Bethesda nomenclature system. Cytology results were considered as the "reference diagnosis" to determine the performance of Self- and Dr-HPV. Two different cutoffs were used to assess the women's disease status. The first cutoff was set at the presence of low-grade squamous intraepithelial lesion or worse (LSIL+), whereas the second cutoff was the presence of high-grade squamous intraepithelial lesion or worse (HSIL+).

\section{Thermoablation}

The procedure consists in the application of a probe heated to $100^{\circ} \mathrm{C}$ on the cervix for $\sim 60$ seconds, which achieves a tissue destruction of 5-7 $\mathrm{mm}$ in depth. An appropriately-sized probe was selected based on the area of the pathological cervical tissue. Several applications were performed, if needed, to cover the entire pathological area.

\section{Data management}

A personalized electronic medical chart including sociodemographic and medical information was created using the secuTrial $^{\circledR}$ (interActive systems, Berlin, Germany) online database to collect and manage data at baseline screening and follow-up.

\section{Statistical analyses}

Data analysis was performed using the Stata Statistical Software Release 14 (StataCorp LP, College Station, TX, USA). Quantitative variables were expressed as mean and SD, and qualitative variables were expressed as percentage, unless otherwise stated. McNemar's test was used to compare the performance of the tests. All tested hypotheses were two sided, and $P$-values $<0.05$ were considered statistically significant.

Sensitivity, specificity, positive predictive value, and negative predictive value (NPV) for the detection of HSIL were calculated for Self-HPV and Dr-HPV, using cytology as the standard reference. Agreement between the Self-HPV and the Dr-HPV was assessed using the $\kappa$ values, and their $95 \%$ CIs were calculated. $\kappa$ values were calculated by obtaining the difference between the observed and the expected agreement and by standardizing the obtained value on $\mathrm{a}-1$ to 1 scale. $\kappa$ values were interpreted according to a commonly used scale. For $\kappa$ values $<0$, the agreement is considered to be less than what would be expected by chance; for $\kappa$ values $0.01-0.20$, only a slight agreement is present; for $\kappa$ values $0.21-0.40$, the agreement is considered to be fair; for $\kappa$ values $0.41-0.60$, the agreement is said to be moderate; for $\kappa$ values $0.61-0.80$, the agreement is considered substantial; and finally, for $\kappa$ values $0.81-0.99$, the agreement is said to be almost perfect. ${ }^{8}$

\section{Ethics approval and informed consent}

This study was approved by the Ethical Cantonal Board of Geneva, Switzerland (Commission Cantonale d'Ethique de la Recherche), with the identification number 15-068. All participants have signed a written informed consent form prior to taking part in the study. All participants have consented to having duplicate analyses of their specimens to fulfill the study's purpose.

\section{Results}

\section{Participants' characteristics}

All of the $188 \mathrm{HPV}$-positive participants were invited to attend the 6 and 12 months control visits. Their mean age was $38.7 \pm 5.6$ years. They had an average of $3.9 \pm 2.8$ sexual partners, and 175/188 (93.1\%) of them were married or in a stable relationship. A total of $116 / 187$ (62.0\%) of the participants had a high-school diploma. The detection rate of CIN grade 2 or worse (CIN 2+) at baseline was 19/1,012 (1.9\%). The participants' sociodemographics and clinical characteristics at baseline are reported in Table 1. Follow-up was pursued for $154 / 188(81.9 \%)$ patients at 6 months and $131 / 188(69.7 \%)$ patients at 12 months.

\section{Performance of Self- and Dr-HPV at 6 months}

Using cytology as the standard reference, Self-HPV had a sensitivity of $84.6 \%$ (95\% CI 49.0-96.9), whereas Dr-HPV had a sensitivity of $92.3 \%$ (95\% CI 53.1-99.2) for the detection of LSIL+ at 6 months $(P=1.000)$. The specificity of Self-HPV for the detection of LSIL+ at 6 months was $68.1 \%$ (95\% CI 59.9-75.3), whereas Dr-HPV had a specificity of 75.7\% (95\% CI 67.8-82.2) ( $P=0.043)$.

The sensitivity of Self-HPV for the detection of HSIL+ at 6 months was 88.9\% (95\% CI 37.4-99.1), whereas Dr-HPV achieved a sensitivity of $100(P=1.000)$. The specificity of Self-HPV for the detection of HSIL+ at 6 months was $66.9 \%$ (95\% CI 58.7-74.1), whereas the specificity of Dr-HPV was $74.3 \%$ (95\% CI 66.5-80.8) ( $P=0.043)$. The performance of Self- and Dr-HPV at 6 months is reported in Table 2.

\section{Performance of Self- and Dr-HPV at 12 months}

Using cytology as the standard reference, Self-HPV had a sensitivity of $85.7 \%$ (95\% CI 25.7-99.0), whereas Dr-HPV 
Table I Sociodemographic and clinical characteristics of the study population

\begin{tabular}{|c|c|}
\hline Variable & n (\%) \\
\hline Age (years), mean $\pm S D$ & $38.7 \pm 5.6$ \\
\hline Age at first sexual intercourse (years), mean \pm SD & $17.9 \pm 2.7$ \\
\hline Gestity, mean \pm SD & $5.0 \pm 2.2$ \\
\hline Parity, mean \pm SD & $4.0 \pm 1.9$ \\
\hline Number of sexual partners, mean $\pm S D$ & $3.9 \pm 2.8$ \\
\hline \multicolumn{2}{|l|}{ Marital status } \\
\hline Single & $13(6.9)$ \\
\hline With a partner & $175(93.1)$ \\
\hline \multicolumn{2}{|l|}{ Education level } \\
\hline None & I $(0.5)$ \\
\hline Elementary school & $39(20.9)$ \\
\hline Apprenticeship & $3(1.6)$ \\
\hline High school & $116(62.0)$ \\
\hline University & $27(14.4)$ \\
\hline Others & I $(0.5)$ \\
\hline \multicolumn{2}{|l|}{ Employment status } \\
\hline Employed & $121(64.4)$ \\
\hline Farmer & $12(6.4)$ \\
\hline Housewife & $48(25.5)$ \\
\hline Others & $7(3.7)$ \\
\hline \multicolumn{2}{|l|}{ Contraception } \\
\hline None & $|4|(75.0)$ \\
\hline Pill & $3(1.6)$ \\
\hline IUD & $4(2.1)$ \\
\hline Injection & $10(5.3)$ \\
\hline Condom & $21(11.2)$ \\
\hline Others & $9(4.8)$ \\
\hline \multicolumn{2}{|l|}{ HPV test result at baseline screening ${ }^{\mathrm{a}}$} \\
\hline HPV-16 & $20(10.7)$ \\
\hline HPV-I8/45 & $42(22.5)$ \\
\hline Other hrHPV & $125(66.8)$ \\
\hline \multicolumn{2}{|l|}{ Histological diagnosis at baseline } \\
\hline Negative & $150(82.9)$ \\
\hline CIN I & $10(5.5)$ \\
\hline $\mathrm{CIN} 2$ & $3(1.7)$ \\
\hline $\mathrm{CIN} 3$ & $15(8.3)$ \\
\hline Invasive carcinoma & $I(0.6)$ \\
\hline \multicolumn{2}{|l|}{ Treatment by thermoablation at baseline } \\
\hline Yes & $12 \mid(64.4)$ \\
\hline No & $67(35.6)$ \\
\hline
\end{tabular}

Notes: CIN 1/2/3, CIN grade 1/2/3. aHPV self-sample test result.

Abbreviations: $\mathrm{CIN}$, cervical intraepithelial neoplasia; HPV, human papillomavirus; hrHPV, high-risk HPV; IUD, intrauterine device; SD, standard deviation. had a sensitivity of $71.4 \%(95 \%$ CI $21.5-95.8)$ for the detection of LSIL+ at 12 months $(P=1.000)$. The specificity of Self-HPV for the detection of LSIL+ at 12 months was $66.1 \%$ (95\% CI 57.2.8-74.0), whereas Dr-HPV had a specificity of $79.0 \%$ (95\% CI 70.9-85.4) ( $P=0.003)$.

The sensitivity of Self-HPV for the detection of HSIL+ at 12 months was $83.3 \%$ (95\% CI 18.6-99.1), whereas Dr-HPV achieved a sensitivity of 71.4 (95\% CI 21.5-95.8) $(P=1.000)$. The specificity of Self-HPV for the detection of HSIL+ at 12 months was $65.6 \%$ (95\% CI 56.7-73.5), whereas the specificity of Dr-HPV was $79.0 \%$ (95\% CI 70.9-85.4) ( $P=0.002)$. The performance of Self- and Dr-HPV at 12 months is reported in Table 3.

\section{Performance of Self- and Dr-HPV according to baseline treatment status}

Among women who underwent thermoablation at baseline screening, the sensitivity of HPV testing using Dr-HPV specimens was identical to that achieved using Self-HPV specimens. Nevertheless, the specificity of Dr-HPV was significantly higher than Self-HPV ( $P=0.003$ ) (Table 4). Among untreated women, data were difficult to interpret because of the lack of power and, therefore, have not been displayed in Table 4. There was no difference between treated and untreated women in terms of the tests' performance.

\section{HPV positivity agreement between Self-HPV and Dr-HPV}

The overall agreement statistics for Self- and Dr-HPV at 6 and 12 months was satisfactory, with a $\kappa$ value of 0.62 and 0.52 , respectively.

$\kappa$ values were stratified by the women's sociodemographic and clinical characteristics. At 6 months, the $\kappa$ value for Self-HPV and Dr-HPV varied between 0.35 and 0.78 . For women positive for HPV-16, the $\kappa$ value was $0.76(95 \%$ CI $0.60-0.92)$. For women aged $40-44$ years, the $\kappa$ value was 0.77 (95\% CI $0.42-1.12)$. At 12 months, the $\kappa$ value

Table 2 Performance of Self-HPV and Dr-HPV at 6 months

\begin{tabular}{|c|c|c|c|c|}
\hline Variable & $\begin{array}{l}\text { Sensitivity, \% } \\
(95 \% \mathrm{Cl})\end{array}$ & $\begin{array}{l}\text { Specificity, \% } \\
(95 \% \mathrm{Cl})\end{array}$ & $\begin{array}{l}\text { PPV, \% } \\
(95 \% \mathrm{Cl})\end{array}$ & $\begin{array}{l}\text { NPV, \% } \\
(95 \% \mathrm{Cl})\end{array}$ \\
\hline \multicolumn{5}{|c|}{ Abnormal cytology LSIL+ } \\
\hline Self-HPV & $84.6(49.0-96.9)$ & $68.1(59.9-75.3)$ & $19.6(11.0-32.6)$ & $98.0(92.0-99.5)$ \\
\hline Dr-HPV & $92.3(53.1-99.2)$ & $75.7(67.8-82.2)$ & $26.1(|5|-4 \mid .2)$. & 99.1 (93.5-99.9) \\
\hline \multicolumn{5}{|c|}{ Abnormal cytology HSIL+ } \\
\hline Self-HPV & $88.9(37.4-99.1)$ & $66.9(58.7-74.1)$ & $14.3(7.1-26.5)$ & $99.0(92.9-99.9)$ \\
\hline Dr-HPV & 100 & $74.3(66.5-80.8)$ & $19.6(10.2-34.1)$ & 100 \\
\hline
\end{tabular}

Abbreviations: HPV, human papillomavirus; HSIL+, high-grade squamous intraepithelial lesion or worse; LSIL+, low-grade squamous intraepithelial lesion or worse; NPV, negative predictive value; PPV, positive predictive value; Self-HPV, HPV test result of the self-taken sample; Dr-HPV, HPV test result of the physician-taken sample. 
Table 3 Performance of Self-HPV and Dr-HPV at 12 months

\begin{tabular}{|c|c|c|c|c|}
\hline Variable & $\begin{array}{l}\text { Sensitivity, \% } \\
\text { (95\% Cl) }\end{array}$ & $\begin{array}{l}\text { Specificity, \% } \\
\text { (95\% Cl) }\end{array}$ & $\begin{array}{l}\text { PPV, \% } \\
(95 \% \mathrm{Cl})\end{array}$ & $\begin{array}{l}\text { NPV, \% } \\
(95 \% \mathrm{Cl})\end{array}$ \\
\hline \multicolumn{5}{|c|}{ Abnormal cytology LSIL+ } \\
\hline Self-HPV & $85.7(25.7-99.0)$ & $66.1(57.2-74.0)$ & $12.5(5.56-25.8)$ & $98.8(91.6-99.8)$ \\
\hline Dr-HPV & $71.4(21.5-95.8)$ & $79.0(70.9-85.4)$ & $16.1(6.5-34.6)$ & $98.0(92.2-99.5)$ \\
\hline \multicolumn{5}{|c|}{ Abnormal cytology HSIL+ } \\
\hline Self-HPV & $83.3(18.6-99.1)$ & $65.6(56.7-73.5)$ & $10.4(4.3-23.3)$ & $98.8(91.6-99.8)$ \\
\hline Dr-HPV & $71.4(21.5-95.8)$ & $79.0(70.9-85.4)$ & I6.I (6.5-34.6) & $99.0(93.0-99.8)$ \\
\hline
\end{tabular}

Notes: Self-HPV, HPV test result of the self-taken sample; Dr-HPV, HPV test result of the physician-taken sample.

Abbreviations: HPV, human papillomavirus; HSIL+, high-grade squamous intraepithelial lesion or worse; LSIL+, low-grade squamous intraepithelial lesion or worse; NPV, negative predictive value; PPV, positive predictive value.

for Self-HPV and Dr-HPV varied between 0.33 and 0.75 . Women positive for other hrHPV types had a $\kappa$ value of 0.63 (95\% CI 0.51-0.79), and women with parity $>5$ had a $\kappa$ value for Self-HPV and Dr-HPV of 0.75 (95\% CI 0.35-1.15). The $\kappa$ values for Self-HPV and Dr-HPV stratified by the women's clinical and sociodemographic characteristics at 6 and 12 months are reported in Figures 1 and 2, respectively.

\section{Discussion}

This study compared the performance of self- and physiciancollected samples for the detection of high-grade cervical lesions at 6 and 12 months following a CC screening campaign. While at 6 month Self-HPV showed a better performance in terms of LSIL+ and HSIL+ detections than Self-HPV, the latter achieved a sensitivity of $85.7 \%$ (95\% CI 25.7-99.0) and 83.3\% (95\% CI 18.6-99.1), for the detection of LSIL+ and HSIL+, respectively, at 12 months, compared to a sensitivity of $71.4 \%$ (95\% CI 21.5-95.8) achieved by Dr-HPV using the two respective cutoffs. Furthermore, the high NPV of the two tests supports the use of these strategies for the exclusion of the disease in the follow-up of HPVpositive women.

Among women treated with thermoablation, we found that Self-HPV was less specific, although the sensitivity between the two strategies (Self- vs Dr-HPV) did not differ significantly. Similarly, Taylor et $\mathrm{al}^{9}$ found that, among women treated with cryotherapy, HPV testing performed on self-collected samples was significantly less specific when compared with HPV testing performed on clinician-collected samples for the detection of CIN. The authors suggest that the lower specificity of Self-HPV may be due to the presence of residual HPV-infected cells in the vagina, despite the clearance of the HPV infection on the cervical transformation zone. Nonetheless, one relevant detail is that HPV testing in the study by Taylor et al was performed using the Hybrid Capture 2 HPV DNA assay, which is known to be less sensitive than the machine GeneXpert we used (81.6 vs $90.8 \%$, $P=0.004) .{ }^{10}$ Speaking in favor of Self-HPV is a prospective study showing that Self-HPV is as accurate as cliniciandirected lavages in describing the natural history of the HPV infection over time. ${ }^{11}$

The $\kappa$ values found in our study show a moderate-tosubstantial agreement between Self-HPV and Dr-HPV when stratified by the women's sociodemographic and clinical characteristics. The fact that the $\kappa$ values do not follow a linear trend according to the participants' characteristics makes our results difficult to interpret. Previous studies have reported a similar agreement rate between self- and physician-collected swabs in primary screening, their $\kappa$-statistic varying between 0.54 and $0.62 .^{12,13}$ Similar to

Table 4 Comparison of Self-HPV and Dr-HPV test performance for the detection of HSIL at I2 months among women treated with thermoablation

\begin{tabular}{|c|c|c|c|c|c|c|}
\hline \multirow[t]{2}{*}{ Variable } & \multicolumn{2}{|l|}{ Cytology } & \multirow{2}{*}{$\begin{array}{l}\text { Sensitivity, \% } \\
(95 \% \mathrm{CI})\end{array}$} & \multirow[t]{2}{*}{$P$-value } & \multirow{2}{*}{$\begin{array}{l}\text { Specificity, \% } \\
\text { (95\% CI) }\end{array}$} & \multirow[t]{2}{*}{$P$-value } \\
\hline & Positive & Negative & & & & \\
\hline Treated women & $n=4$ & $\mathrm{n}=82$ & & & & \\
\hline \multicolumn{7}{|l|}{ Self-HPV } \\
\hline Positive & 3 & 28 & $75.0(28.9-96.6)$ & 1.000 & $65.9(55.1-75.2)$ & $0.003^{*}$ \\
\hline Negative & 1 & 54 & & & & \\
\hline \multicolumn{7}{|l|}{ Dr-HPV } \\
\hline Positive & 3 & 14 & $75.0(28.9-96.6)$ & & $82.9(73.2-89.7)$ & \\
\hline Negative & 1 & 68 & & & & \\
\hline
\end{tabular}

Notes: Self-HPV, HPV test result of the self-taken sample; Dr-HPV, HPV test result of the physician-taken sample. $* P$-value $<0.05$. 


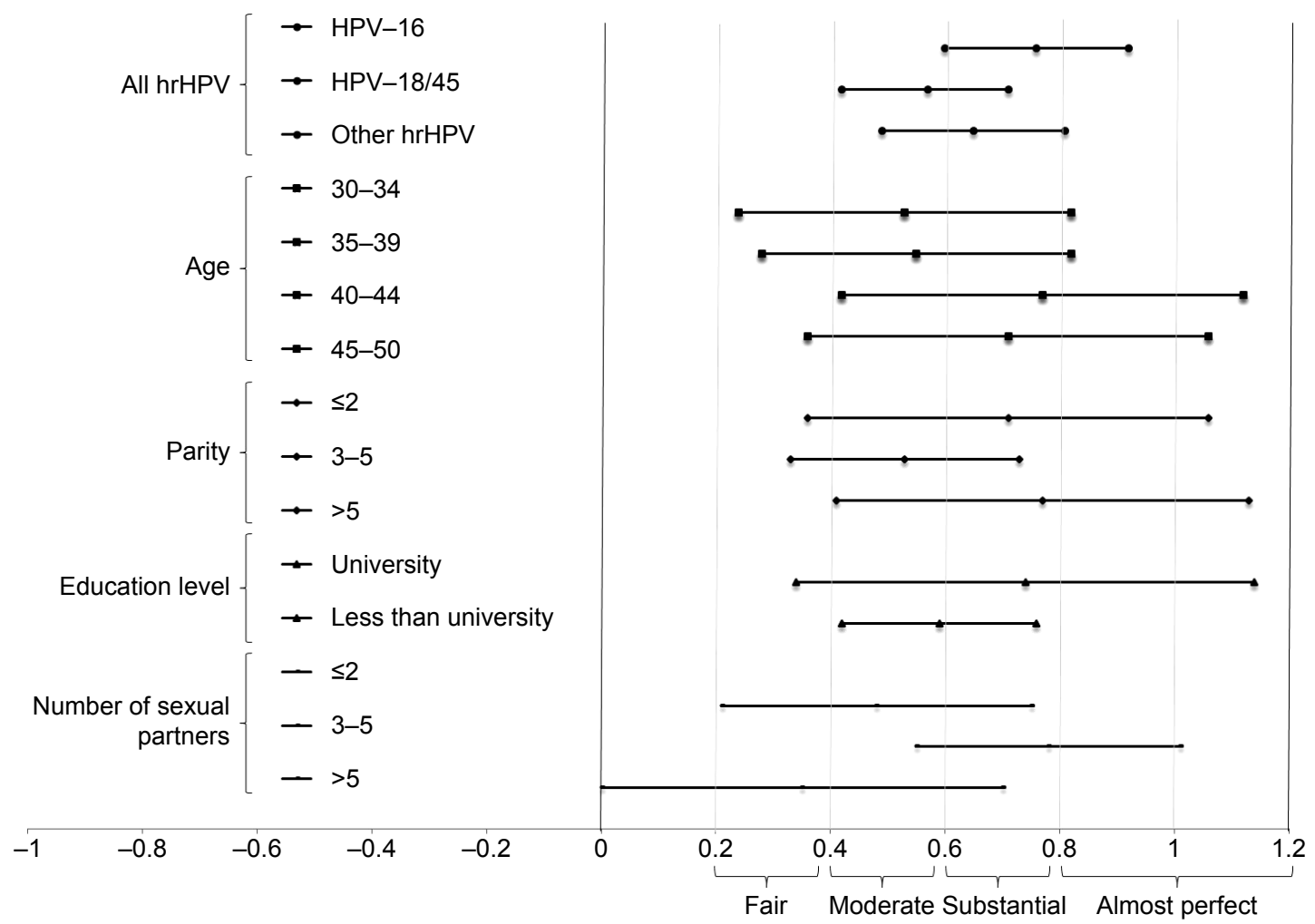

Figure I Agreement statistics of HPV positivity ( $\kappa$ value and $95 \% \mathrm{Cl}$ ) for Self-HPV and Dr-HPV according to patient characteristics at 6 months. Notes: Self-HPV, HPV test result of the self-taken sample; Dr-HPV, HPV test result of the physician-taken sample.

Abbreviations: HPV, human papillomavirus; hrHPV, high-risk HPV.

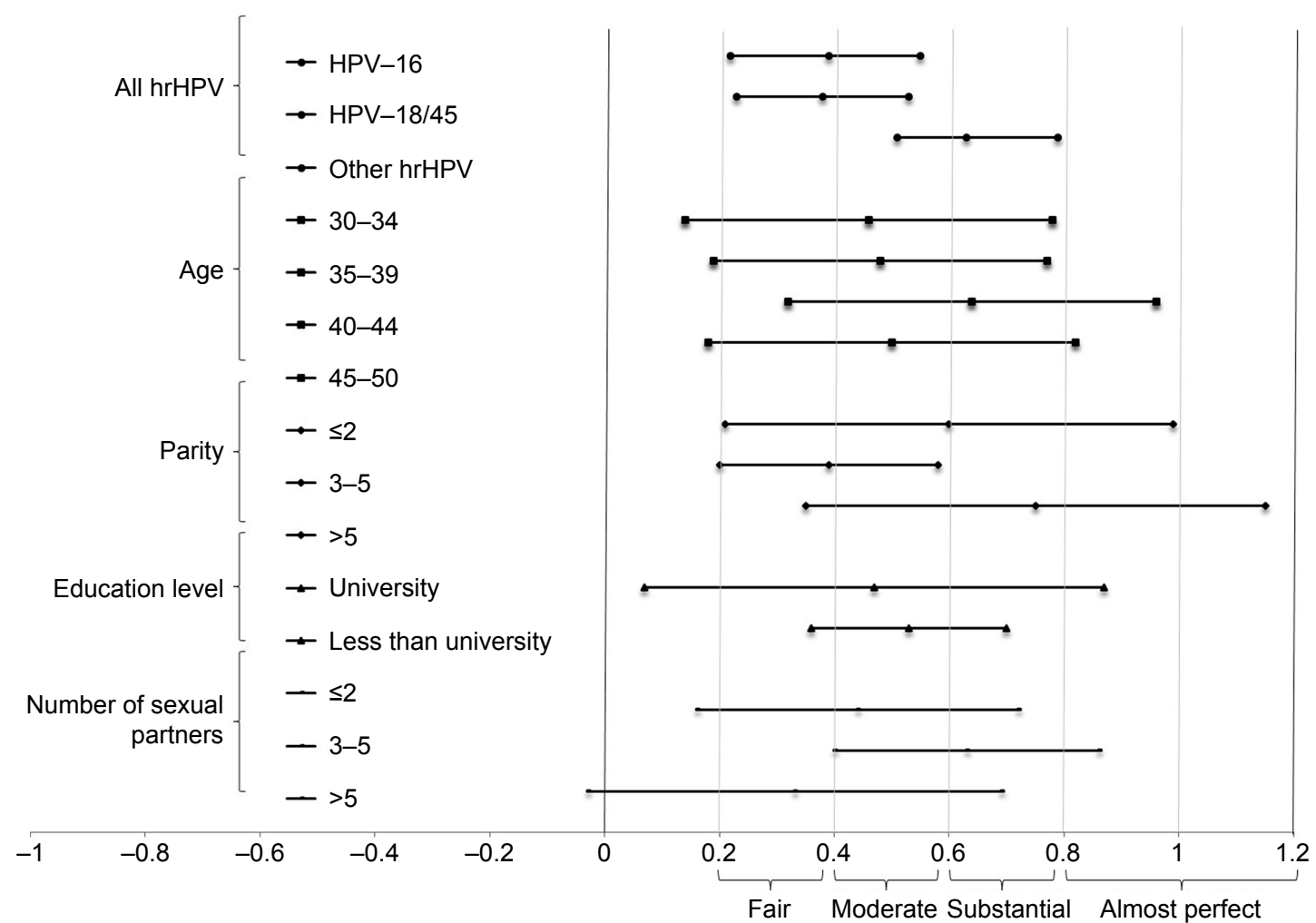

Figure 2 Agreement statistics of HPV positivity ( $\kappa$ value and $95 \% \mathrm{Cl}$ ) for Self-HPV and Dr-HPV according to patient characteristics at 12 months. Notes: Self-HPV, HPV test result of the self-taken sample; Dr-HPV, HPV test result of the physician-taken sample.

Abbreviations: HPV, human papillomavirus; hrHPV, high-risk HPV. 
Johnson et al, we obtained a substantially improved agreement between Self- and Dr-HPV test results for women who tested positive for HPV-16 (97.7\%). ${ }^{13,14}$

We found that Self-HPV had a lower specificity among treated women. This issue could be solved by pairing SelfHPV with either cytology or VIA/VILI for the follow-up of previously treated women. As a previous cervical treatment status dictates the necessity to undergo a gynecological examination, in such context, Self-HPV could serve as a supplementary tool, rather than a substitute of the pelvic examination-based strategies. Increasing evidence has shown that HPV testing used on women treated with ablative techniques, such as cryotherapy, vaunts a high sensitivity and an NPV for the detection of residual or recurrent disease. ${ }^{14}$ In a study comparing different follow-up techniques for women treated with cryotherapy, Omenge Orang'o et al ${ }^{15}$ demonstrated that, although at the price of a lower specificity when compared with cytology and VIA, HPV testing alone has the best sensitivity for the detection of CIN $2+$.

A recently published review has concluded that selfsampling is generally well accepted by women. ${ }^{16}$ The main reasons for the better acceptance of Self-HPV compared to Dr-HPV are its ease of use and respect of the women's intimacy. ${ }^{16}$ This high acceptance could potentially reduce the loss to follow-up that is currently responsible for low program impact in developing countries. ${ }^{17}$

One strength of our study is that all patients benefited from cytology at 6 and 12 months, thus allowing us to validate the performance of the two tests using cytology as the reference diagnosis. To our knowledge, this is one of the first studies to compare the performance of Self- and Dr-HPV for the follow-up of screen-positive women with that of women treated by thermoablation in a low-resource setting.

Limitations that need to be addressed are the small sample size and the $30.3 \%$ loss to follow-up rate at 12 months, which limits the generalization of our findings to the rest of the population. Larger, prospective cohort studies are needed to confirm our results by comparing them directly with other follow-up strategies, such as VIA/VILI and cytology. Another limitation is the discrepancy between the use of $\mathrm{NaCl} 0.9 \%$ and the SurePath medium for HPV analysis of Self-HPV and Dr-HPV, respectively. Our previous experience has demonstrated that the use of $\mathrm{NaCl} 0.9 \%$ is a valid medium for HPV analysis on Self-HPV. ${ }^{18}$ The use of this medium, however, has not yet been proven effective for Dr-HPV. Therefore, the $\mathrm{NaCl}$ 0.9\% was used for Self-HPV sample analysis, whereas the SurePath medium, as recommended by the manufacturers, was used for the analysis of Dr-HPV samples.

\section{Conclusion}

The use of HPV testing on self-collected samples is a valuable option for the follow-up of HPV-positive women in LMIC. Although the specificity of Dr-HPV is slightly superior, the advantages of Self-HPV seem to outweigh its flaws, making it a valuable tool to break down the main barrier to follow-up attendance in LMIC.

\section{Data availability}

The data are securely stored in the online database SecuTrial. Data will be available upon request.

\section{Acknowledgments}

The authors would like to thank Dr Mohamed Akaaboune, who passed away on July 31, 2017, for his precious contribution to the project. His two trips to Cameroon, his silent efforts, and his meticulous data collection have made the present project's realization possible. This study was supported by the Dschang District Hospital, Cameroon, and the University Hospitals of Geneva, Switzerland. The funders had no role in study design, data collection and analysis, decision to publish, or preparation of the article.

\section{Disclosure}

The authors report no conflicts of interest in this work.

\section{References}

1. Ferlay J, Soerjomataram I, Dikshit R, et al. Cancer incidence and mortality worldwide: sources, methods and major patterns in GLOBOCAN 2012. Int J Cancer. 2015;136(5):E359-E386.

2. Bae J, Seo SS, Park YS, et al. Natural history and epidemiology of HPV infection and cervical cancer. Gynecol Oncol. 2009;115(1):75-80.

3. Aerssens A, Claeys P, Beerens F, et al. Prediction of recurrent disease by cytology and HPV testing after treatment of cervical intraepithelial neoplasia. Cytopathology. 2009;20(1):27-35.

4. Rattanalappaiboon D, Kleebkaow $P$, Chumworathayi B, Launratanakom S, Santipongsupakorn T. Factors affecting compliance in the first year of postcolposcopy surveillance among women with a high incidence of cervical cancer. Int J Gynaecol Obstet. 2014;124(2):160-163.

5. Sandri MT, Lentati P, Benini E, et al. Comparison of the Digene HC2 assay and the Roche AMPLICOR human papillomavirus (HPV) test for detection of high-risk HPV genotypes in cervical samples. $J$ Clin Microbiol. 2006;44(6):2141-2146.

6. Arbyn M, Verdoodt F, Snijders PJ, et al. Accuracy of human papillomavirus testing on self-collected versus clinician-collected samples: a meta-analysis. Lancet Oncol. 2014;15(2):172-183.

7. Arbyn M, Herbert A, Schenck U, et al. European guidelines for quality assurance in cervical cancer screening: recommendations for collecting samples for conventional and liquid-based cytology. Cytopathology. 2007;18(3):133-139.

8. Viera AJ, Garrett JM. Understanding interobserver agreement: the kappa statistic. Fam Med. 2005;37(5):360-363.

9. Taylor S, Wang C, Wright TC, Denny L, Kuhn L. A comparison of human papillomavirus testing of clinician-collected and self-collected samples during follow-up after screen-and-treat. Int J Cancer. 2011;129(4) 879-886. 
10. Einstein MH, Smith KM, Davis TE, et al. Clinical evaluation of the cartridge-based GeneXpert human papillomavirus assay in women referred for colposcopy. J Clin Microbiol. 2014;52(6):2089-2095.

11. Moscicki AB, Widdice L, Ma Y, et al. Comparison of natural histories of human papillomavirus detected by clinician- and self-sampling. Int J Cancer. 2010;127(8):1882-1892.

12. Johnson DC, Bhatta MP, Smith JS, et al. Assessment of high-risk human papillomavirus infections using clinician- and self-collected cervical sampling methods in rural women from far western Nepal. PLoS One. 2014;9(6):e101255.

13. Ting J, Mugo N, Kwatampora J, et al. High-risk human papillomavirus messenger RNA testing in physician- and self-collected specimens for cervical lesion detection in high-risk women, Kenya. Sex Transm Dis. 2013;40(7):584-589.

14. Verguts J, Bronselaer B, Donders G, et al. Prediction of recurrence after treatment for high-grade cervical intraepithelial neoplasia: the role of human papillomavirus testing and age at conisation. BJOG. 2006; 113(11):1303-1307.
15. Omenge Orang'o O, Liu T, Christoffersen-Deb A, et al. Use of visual inspection with acetic acid, Pap smear, or high-risk human papillomavirus testing in women living with HIV/AIDS for posttreatment cervical cancer screening: same tests, different priorities. AIDS. 2017;31(2): 233-240.

16. Nelson EJ, Maynard BR, Loux T, Fatla J, Gordon R, Arnold LD. The acceptability of self-sampled screening for HPV DNA: a systematic review and meta-analysis. Sex Transm Infect. 2017;93(1):56-61.

17. Petignat P, Faltin DL, Bruchim I, Tramer MR, Franco EL, Coutlee F. Are self-collected samples comparable to physician-collected cervical specimens for human papillomavirus DNA testing? A systematic review and meta-analysis. Gynecol Oncol. 2007;105(2):530-535.

18. Kunckler M, Schumacher F, Kenfack B, et al. Cervical cancer screening in a low-resource setting: a pilot study on an HPV-based screen-and-treat approach. Cancer Med. 2017;6(7):1752-1761.

\section{Publish your work in this journal}

The International Journal of Women's Health is an international, peerreviewed open-access journal publishing original research, reports, editorials, reviews and commentaries on all aspects of women's healthcare including gynecology, obstetrics, and breast cancer. The manuscript management system is completely online and includes

\section{Dovepress}

a very quick and fair peer-review system, which is all easy to use Visit http://www.dovepress.com/testimonials.php to read real quotes from published authors. 